\title{
Telomerase-positive circulating tumor cells are associated with poor prognosis via a neutrophil-mediated inflammatory immune environment in glioma
}

Wen Zhang ${ }^{1 \dagger}$, Tiancheng Qin ${ }^{2 \dagger}$, Zhenrong Yang ${ }^{2}$, Liyuan Yin ${ }^{3}$, Changyun Zhao ${ }^{4}$, Lin Feng ${ }^{2}$, Song Lin ${ }^{5,6^{*}}$, Binlei Liu ${ }^{7^{*}}$, Shujun Cheng ${ }^{2}$ and Kaitai Zhang ${ }^{2^{*}}$

\begin{abstract}
Background: Gliomas are the most common aggressive cancer in the central nervous system. Considering the difficulty in monitoring glioma response and progression, an approach is needed to evaluate the progression or survival of patients with glioma. We propose an application to facilitate clinical detection and treatment monitoring in glioma patients by using telomerase-positive circulating tumor cells (CTCS) and to further evaluate the relationship between the immune microenvironment and CTCs in glioma patients.

Methods: From October 2014 to June 2017, 106 patients newly diagnosed with glioma were enrolled. We used the telomerase reverse transcriptase CTC detection method to detect and analyze the CTC statuses of glioma patients before and after surgery. FlowSight and FISH confirmed the CTCS detected by the telomerase-based method. To verify the correlation between CTCs and the immune response, peripheral white blood cell RNA sequencing was performed.
\end{abstract}

\footnotetext{
*Correspondence: linsong2005@126.com; liubl@hbut.edu.cn; zhangkt@cicams.ac.cn

'Wen Zhang and Tiancheng Qin contributed equally to this work.

${ }^{5}$ Department of Neurosurgery, Beijing Tiantan Hospital, Capital Medical University, Beijing, China

${ }^{7}$ National "111" Center for Cellular Regulation and Molecular Pharmaceutics, Key Laboratory of Fermentation Engineering (Ministry of Education), Hubei Provincial Cooperative Innovation Center of Industrial Fermentation, College of Bioengineering, Hubei University of Technology, Wuhan 430068, China ${ }^{2}$ State Key Laboratory of Molecular Oncology, Department of Etiology and Carcinogenesis, National Cancer Center/National Clinical Research Center for Cancer/Cancer Hospital, Chinese Academy of Medical Sciences and Peking Union Medical College, Beijing 100021, China

Full list of author information is available at the end of the article
}

(c) The Author(s). 2021 Open Access This article is licensed under a Creative Commons Attribution 4.0 International License, which permits use, sharing, adaptation, distribution and reproduction in any medium or format, as long as you give appropriate credit to the original author(s) and the source, provide a link to the Creative Commons licence, and indicate if changes were made. The images or other third party material in this article are included in the article's Creative Commons licence, unless indicated otherwise in a credit line to the material. If material is not included in the article's Creative Commons licence and your intended use is not permitted by statutory regulation or exceeds the permitted use, you will need to obtain permission directly from the copyright holder. To view a copy of this licence, visit http://creativecommons.org/licenses/by/4.0/. The Creative Commons Public Domain Dedication waiver (http://creativecommons.org/publicdomain/zero/1.0/) applies to the data made available in this article, unless otherwise stated in a credit line to the data. 
Results: CTCs were common in the peripheral blood of glioma patients and were not correlated with the pathological classification or grade of patients. The results showed that the presence of postoperative CTCs but not preoperative CTCs in glioma patients was a poor prognostic factor. The level of postoperative CTCs, which predicts a poor prognosis after surgery, may be associated with neutrophils. RNA sequencing suggested that postoperative CTCS were positively correlated with innate immune responses, especially the activation of neutrophils and the generation of neutrophil extracellular traps, but negatively correlated with the cytotoxic response.

Conclusions: Our results showed that telomerase-positive CTCS can predict a poor prognosis of patients with glioma. Our results also showed a correlation between CTCS and the immune macroenvironment, which provides a new perspective for the treatment of glioma.

Keywords: Circulating tumor cells, Telomerase, Prognosis, Glioma, Neutrophils, Immune macroenvironment, Neutrophil extracellular traps

\section{Background}

Glioma is the most common aggressive cancer in the central nervous system (CNS) and represent $75 \%$ of primary intracranial tumors in adults [1]. Many previous studies have shown that after surgery combined with radiotherapy and chemotherapy, glioma still has an aggressive tendency toward intracranial recurrence, and the growth rate after recurrence is accelerated, worsening patient prognosis $[2,3]$. Data have shown that the median recurrence period of glioblastoma (GBM) is 812 months, the median recurrence period of stromal glioma is $18-36$ months, and the median overall survival (OS) of recurrent glioma is only 25-35 weeks [4, 5]. Therefore, considering the poor prognosis of gliomas and the difficulty in monitoring glioma response and progression, it is necessary to establish an approach for evaluating the progression or survival of patients with glioma.

Advancement in the development of molecular biology techniques for glioma has led to the discovery of a series of biomarkers with practical value for diagnosis, differential diagnosis, and treatment [6, 7]. Although these molecular markers have made great breakthroughs in the diagnosis and treatment of glioma, patient prognosis is still not optimistic, and there is no molecular biomarker that can accurately distinguish the subtypes of glioma or predict prognosis. The rapid development of liquid biopsy technology, especially relevant studies on circulating tumor cells (CTCs), shows that this technology is helpful for the clinical treatment and prognosis prediction of GBM patients $[8,9]$. Although CTC studies in glioma patients show potential clinical applications, there are still some questions that need to be answered, such as whether CTCs can represent the main nature of tumor cells to correctly reflect the clinical behavior of GBM patients with recurrence and metastasis and provide more accurate information for individual treatment and prognosis prediction.
Based on previous studies $[10,11]$, a viable CTC detection method based on human telomerase reverse transcriptase (TBCD) was used in this study to evaluate the preoperative and postoperative CTC status of glioma patients. Next, the correlation between CTCs and clinical indicators was studied, and recurrence and prognosis were assessed. To better illustrate the clinical application value of CTCs in glioma, we analyzed the significance of the correlation between CTCs and patients' macroimmunity in depth and proposed that the postoperative macroimmunity status of patients is closely related to CTCs and patient prognosis. To the best of our knowledge, this study is the first on the correlation between macroimmunity and CTCs in glioma patients, and we believe that our results can provide a new perspective for the clinical application of CTCs in glioma and even the treatment of glioma.

\section{Methods \\ Collection of patient and clinical information}

In this study, patients and healthy control volunteers all provided written informed consent, and peripheral blood collections were approved by the Ethics Committee of the Cancer Hospital, Chinese Academy of Medical Sciences. From October 2014 to June 2017, 106 patients newly diagnosed with glioma were enrolled, and the clinical information of the included patients was provided by the Department of Neurosurgery, Beijing Tiantan Hospital. Furthermore, another 31 patients were enrolled according to the screening conditions for RNA sequencing research. The clinical characteristics of the enrolled patients are shown in Table 1 . All methods were performed in accordance with the approved guidelines.

\section{Blood samples}

To explore the significance of tumor reduction surgery on CTCs of glioma, we detected the patients' CTC counts before surgery and compared them with their CTC counts at 7 days post-operation. All patients with 
Table 1 Patient Characteristics

\begin{tabular}{ll}
\hline No. of patients & 106 \\
\hline Age (yr) & \\
Mean (range) & $42.63(13-70)$ \\
Sex (\%) & \\
Male & $37(34.9)$ \\
Female & $69(65.1)$ \\
Pathology (\%) & \\
A & $19(17.9)$ \\
O & $5(4.7)$ \\
AO & $8(7.5)$ \\
OA & $29(27.4)$ \\
AOA & $15(14.2)$ \\
GBM & $30(28.3)$ \\
WHO Grade (\%) & \\
1 & $2(1.9)$ \\
2 & $37(34.9)$ \\
3 & $31(29.2)$ \\
4 & $36(34.0)$ \\
KPS (\%) & \\
70 & $2(1.8)$ \\
100 & $10(9.5)$ \\
\hline 0 & $25(23.6)$ \\
\hline 0 & $66(62.3)$ \\
\hline 0 & $3(2.8)$ \\
\hline
\end{tabular}

$A$ astrocytoma; $O$ oligodendroglioma; $A O$ anaplastic oligodendroglioma; $O A$ oligoastrocytoma; $A O A$ anaplastic oligoastrocytoma; $G B M$ glioblastoma multiforme; KPS Karnofsky performance status.

gliomas were first treated and underwent surgery, and long-term follow-up was conducted after the surgery. Four milliliters of blood was collected from eligible patients or healthy donors (aged 20-50 years) in K2E (EDTA) tubes and stored at $4{ }^{\circ} \mathrm{C}$ in the lab within $2 \mathrm{~h}$. The peripheral blood of another 31 glioma patients was collected for WBC RNA sequencing, and postoperative CTCs were also detected.

\section{Cell and virus agent}

The human glioma cell line U251 was obtained from the Cell Resource Center, Peking Union Medical College, and cultured using minimum essential medium with Earle's balanced salts (MEM-EBSS) containing 10\% fetal bovine serum (FBS) at $37{ }^{\circ} \mathrm{C}$ in $5 \% \mathrm{CO}_{2}$.

In this study, the oHSV1-hTERT-GFP virus was constructed as described in a previous study [10]. Based on the selectivity of telomere activity, the oHSV1-hTERTGFP virus captured telomere activity that is widely expressed in cancer cells and expressed green fluorescent protein (GFP).

\section{Identification of samples with glioma}

The peripheral blood of patients with glioma was collected $(4 \mathrm{ml})$ and prepared with $\mathrm{K}_{2}$ E-EDTA anticoagulant tubes within $2 \mathrm{~h}$ of isolation. Details of the TBCD that we used have been described previously $[10,11]$. APC-anti-human CD45 (clone: HI30, Invitrogen, USA) was added to $10^{7}$ cells/sample and incubated at room temperature for $30 \mathrm{~min}$. Flow cytometry (BD, USA) was used to detect the GFP+/CD45- cell population, which was recorded as a positive result (Additional file 1: Fig S1A).

\section{GFP fluorescence observation}

U251 cells or patient samples were transfected with the viral agent and cultured in a 6-well plate for $24 \mathrm{~h}$, and the cells were observed under a fluorescence microscope (Olympus).

\section{Fluorescent in situ hybridization (FISH)}

FISH was performed using a Vysis LSI 1p36 Microdeletion Region Probe (Abbott). DNA-FISH was applied to investigate the presence of CTCs. Peripheral blood samples $(4 \mathrm{ml})$ from glioma patients were treated with a standard CTC testing procedure with some modifications. Peripheral blood samples were transfected with oHSV1-hTERT-GFP and cultured for $24 \mathrm{~h}$. The cells were selected with an MS column by using anti-CD45 microbeads. The unlabeled cells were collected on a slide, and the slides were observed under a fluorescence microscope, confirming that the GFP+ cells were on the slide. Probes were used according to the manufacturer's protocol. The prepared slides were scanned by fluorescence in situ scanner.

\section{Identification of CTCs using ImageStreamX ${ }^{\circledast}$}

APC-CD56 antibody (clone: CMSSB, Invitrogen, USA) and Alexa Fluor 405 CD45 antibody (clone: HI30, Invitrogen, USA) were used to identify CTCs. The CTC images were detected by the ImageStreamX ${ }^{\circ}$ Mark II system (Amnis) (Additional file 1: Fig S1B). CD45/ GFP+/CD56+ cells in the blood samples were considered CTC-positive cells.

\section{Next-generation sequencing (RNA-seq) and data processing}

Peripheral blood was obtained from healthy controls $(n=$ $23)$ and untreated glioma patients $(n=31)$. After red blood cell lysis buffer (Qiagen) was used to remove red blood cells, the total RNA of the remaining WBCs was extracted using TRIzol (Invitrogen). RNA-seq libraries were prepared from qualified samples using the NEBNext $^{\circ}$ Ultra $^{\mathrm{Tm}}$ RNA Library Prep Kit for Illumina (NEB) according to the manufacturer's instructions. 
Clean reads in FASTQ files were quantified against an Ensembl catalog (GRCh37) at the transcript level using Salmon and aggregated to the gene level using tximport; transcripts per million reads (TPM) values were obtained with Salmon gene expression quantification software, and unexpressed genes were removed.

We defined genes positively correlated with CTCs as having a correlation coefficient $R>0.4, p<0.05$ (Cytoscape software) [12], which presented biological role specificity for the most significant differentially expressed gene transcripts based on their functional and pathway enrichment probes. The GO database [13] was used. Immune cells with differentially expressed genes were distinguished by using the "DESeq2" $\mathrm{R}$ package; genes with fold change $>2$ and $p<0.05$ were defined as differentially expressed genes. Heatmaps were made by using the "Pheatmap" $\mathrm{R}$ package, and survival curves were evaluated with the log-rank test (two-tailed) by the Kaplan-Meier method. GO analysis and hierarchy relation analysis were conducted with the "topGO" $\mathrm{R}$ package.

For each given gene list, pathway and process enrichment analyses were carried out using Metascape [14] with the following ontology sources: KEGG Pathway and GO Biological Processes. Z-score analysis was conducted with the "GOplot" R package. All genes in the genome were used as the enrichment background. Terms with a $p$ value $<0.01$, a minimum count of 3 , and an enrichment factor $>1.5$ (the enrichment factor is the ratio between the observed counts and the counts expected by chance) were collected and grouped into clusters based on their membership similarities.

GSEA was performed using the "GSVA" package, which uses the $\mathrm{C} 7$ immunologic signature gene sets and Hallmark gene sets downloaded from the Molecular Signatures Database (MSigDB) $[15,16]$. Estimating the Proportion of Immune and Cancer cells (EPIC) [17] was used to analyze peripheral blood cell distribution.

GSEA enrichment analysis between immune-related gene sets and NETS was conducted by enrichment score in peripheral blood genes compared with different CTCS levels in the two groups. The immune gene set was also derived from a publication published by Bindea G. et al. [18]. Using the gene expression signatures of NETs, the GSEA implementation method relies on the $\mathrm{R}$ package "GSEABase," resulting in an enrichment score ch gene set and NE values for the immune-related phenotypes of the 20 peripheral blood transcriptomes generated using the above methods.

\section{Statistics}

The data were analyzed by generalized linear regression ANOVA in a cell line model and clinical samples. The cutoff value was determined by the Youden index and
ROC curve. The Spearman correlation coefficient was used to assess correlations with clinical information. The Kaplan-Meier method was used to estimate the event time distribution, and the log-rank test was used for comparison. A multivariate Cox proportional hazards regression model was used to check the clinical descriptive information. SPSS 24.0 and GraphPad Prism 7.0 were used for data analysis. All the statistical tests were twosided, with $\mathrm{p}$ values of less than 0.05 considered significant.

\section{Results}

TBCD effectively detected glioma CTCs in glioma patients Previous studies have shown that TBCD can effectively detect CTCs in patients with different types of tumors. Therefore, we first verified the effectiveness and accuracy of this method in detecting glioma patients' CTCs in vitro. As shown in Additional file 1: Fig S2A, the U251 cells expressed GFP $12 \mathrm{~h}$ after oHSV1-hTERTGFP transduction, and a strong positive signal for GFP was detected in almost all U251 cells at $24 \mathrm{~h}$ after transduction. Variable numbers of U251 cells were spiked into 4-ml blood samples from healthy donors and analyzed using TBCD to confirm the accuracy. The recovery rate of U251 cells was $67.5-85.7 \%$. The number of CD45-/GFP+ cells and the number of glioma cells that spiked in showed a strong correlation $\left(r^{2}=0.9871,95 \%\right.$ CI, 0.7213 to 0.7767 ) (Additional file 1 Figure: S2B). This result suggested that this method can reflect the number of CTCs in the in vitro model well.

Then, we analyzed 106 whole blood samples from patients with different stages of glioma aged 13 to 79 years (mean $=42.63$ years). The details of the patient characteristics for CTC detection are listed in Table 1. Based on the ROC analysis of CTC data from 52 healthy donors and 106 patients with glioma (Fig. 1A), the AUC of this model was 0.892 (95\% CI, 0.843-0.941). The threshold was the cutoff point with the Youden index (sensitivity $=0.83$, specificity $=0.865$ ), defined as 1 CTC per $4 \mathrm{ml}$ of blood (Fig. 1B). The average number of detected CD45 $-/$ GFP + cells was $8.453 \pm 0.955$ cells $/ 4 \mathrm{ml}$ for patients with glioma, while the average number of detected CD45-/GFP+ cells was $0.615 \pm 0.107$ cells $/ 4 \mathrm{ml}$ for healthy donors in the control group $(p<0.0001$, Additional file 1 Figure: S3).

Then, circulating glioma cells were imaged using green fluorescence (Fig. 1C). The results showed that CTCs existed in the peripheral blood and could be detected by TBCD. One of the most widely used markers for glioma is $1 \mathrm{p} / 19 \mathrm{q} \mathrm{LOH}$, and we used FISH to visualize CTCs. The specimens we detected had the typical $1 \mathrm{p}$ deletion (Fig. 1D). To ensure that there were no false-positive cells from the WBCs in the sample, we used TBCD for clinical blood samples subjected to flow imaging. 


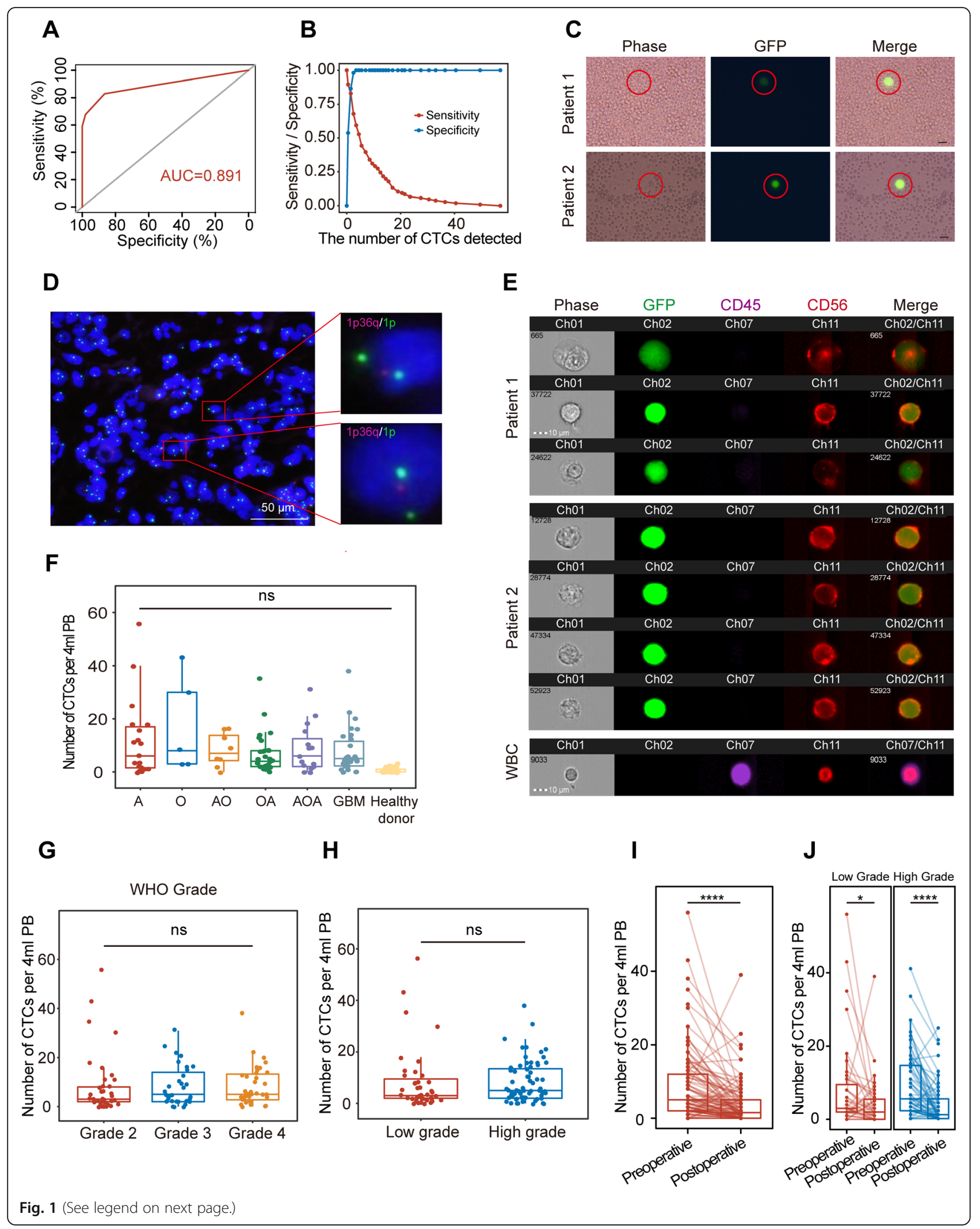


(See figure on previous page.)

Fig. 1 TBCD effectively detected glioma CTCs in glioma patients. A ROC analysis for the threshold detection of CTCs (AUC=0.892). B Youden index analysis of TBCD according to previous findings in the group $(n=158)$ with 52 healthy donors and 106 glioma patients. C. Typical CTCs in the peripheral blood leukocytes of the blood sample were visualized using GFP expression. Scale bar, $10 \mu \mathrm{m}$. D Images of CTCs in GBM patients revealed by DNA-FISH. Red: 1p; Green: 1q. Scale bar, 50 um. E Typical CTC images of ImageStream ${ }^{\circledast}$ flow cytometry analysis. Ch01: bright field; Ch02: GFP (green); Ch07: CD45 (purple); Ch11: CD56 (red). CTCs were identified as CD45 /GFP $/ \mathrm{CD}^{+} 6^{+}$or CD45 $/ \mathrm{GFP}^{+} / \mathrm{CD}^{-} 6^{-}$, and WBCs were $\mathrm{CD}_{5} 5^{+} / \mathrm{CD} 56^{+}$. Scale bar, $10 \mu \mathrm{m}$. F Number of CTCs in 6 pathologic types of gliomas. A, astrocytoma; O, oligodendroglioma; $\mathrm{AO}$, anaplastic oligodendroglioma; OA, oligoastrocytoma; AOA, anaplastic oligoastrocytoma; GBM, glioblastoma multiforme. G Comparison of CTC counts in grade II ( $n=36)$, grade III ( $n=26)$, and grade IV ( $n=26)$ gliomas. H Comparison of CTC counts between low-grade (WHO grades 1 and $2, n=38)$ and high-grade (WHO grades 3 and 4, $n=52$ ) gliomas. I The number of CTCs detected in preoperative and postoperative patients. ${ }^{* * * *}, p<0.0001$. J The number of CTCS detected in the low-grade group and the high-grade group. *, $p=0.044 ; * * *, p<0.0001$

Antibodies against CD45 and CD56 were used to identify cancer cells [19]. As shown in Fig. 1E, GFP+/CD45 $-/ \mathrm{CD} 56+$ cells were identified as glioma cells, and CD45+/CD56+ cells were identified as NK cells (Additional file 1 Figure: S4). These results indicate that TBCD can efficiently and accurately detect CTCs in glioma patients.

\section{CTCs were not related to the clinical features of glioma patients}

As shown in Table 1, CTCs were found in all 6 pathologic subtypes of gliomas, including astrocytoma (A), oligodendroglioma $(\mathrm{O})$, anaplastic oligodendroglioma $(\mathrm{AO})$, oligoastrocytoma (OA), anaplastic oligoastrocytoma (AOA), and GBM. Based on the results of ROC analysis, we defined patients with a test value of $\leq 1$ cell/ $4 \mathrm{ml}$ as a negative result. The total incidence of CTCs was $83.02 \%$ (88/106) before surgery. The variation in preoperative CTC counts ranged from 0 to 56 per $4 \mathrm{ml}$ of peripheral blood (Additional file 2). However, no significant difference in CTC counts was observed between different pathologic subtypes of gliomas (Fig. 1F$\mathrm{H})$. The positive rate of preoperative CTCs ranged from $62.5 \%$ to $100 \%$ (Additional file 1 Figure: S5). However, there were no significant differences between different grades in preoperative CTC counts (Additional file 3). These results suggested that CTCs were widely present in the peripheral blood of glioma patients, and their count was not related to the pathological classification or grade of patients before surgery.

Subsequently, we compared the changes in CTCs before and after surgery in glioma patients. Notably, the CTC counts of patients before and after surgery were significantly different $(p<0.0001)$ (Fig. 1I), but there was no correlation between preoperative CTCs and postoperative CTCs (Pearson $r=0.3642$ ). As shown in Fig. 1J, the CTC counts of the high-grade group and low-grade group were significantly different $(p<0.0001$ and $p=$ 0.044 , respectively) preoperatively and postoperatively. However, as shown in Fig. 2A and B, there were also no

\section{A}

\section{Preoperative CTCs}

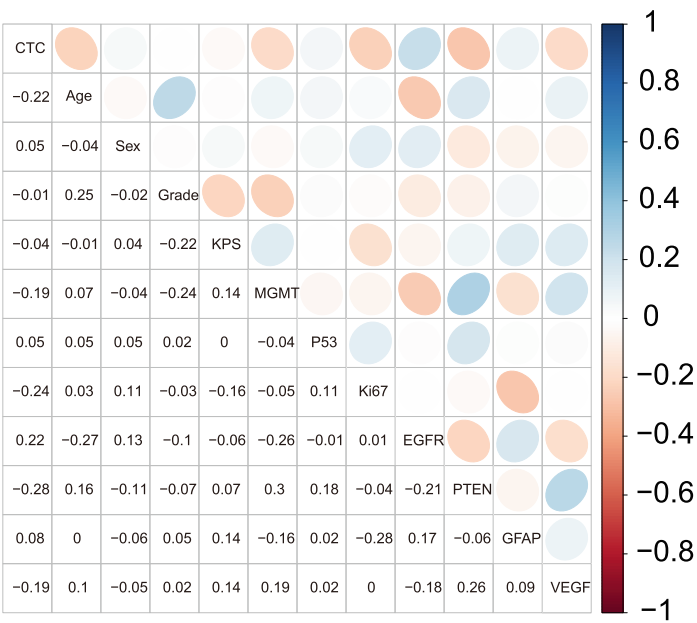

\section{B}

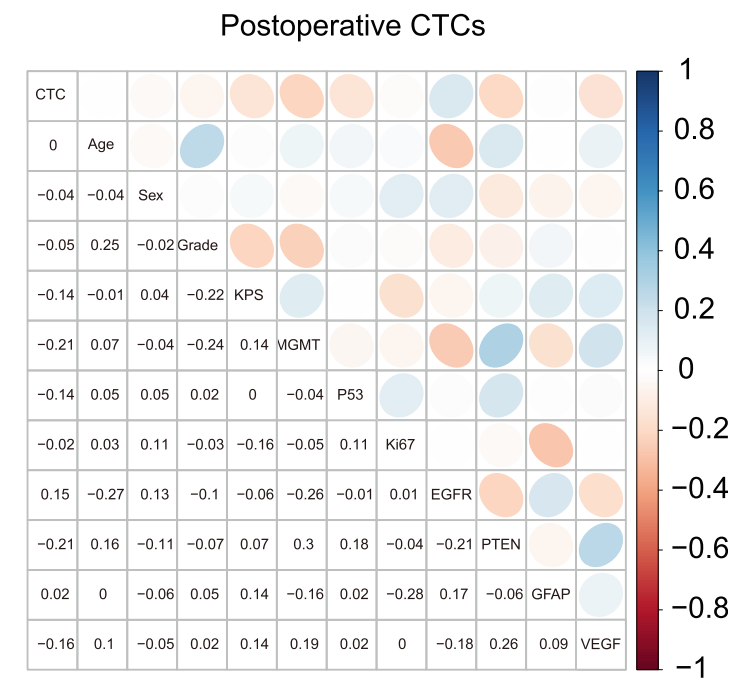

Fig. 2 Postoperative CTCS as a prognostic biomarker. A Correlation heatmap (Pearson correlation) of CTCs and clinical parameters. Positive correlations are shown in cool colors, while negative correlations (anticorrelations) are shown in hot colors. B Correlation heatmap of postoperative CTCs and clinical parameters. Positive correlations are shown in cool colors, while negative correlations (anticorrelations) are shown in hot colors 
correlations between preoperative/postoperative CTCs and patient clinical features or tumor markers (MGMT, P53, Ki67, EGFR, PTEN, GFAP, and VEGF). These results suggested that the presence of CTCs was independent of patient and tumor characteristics.

\section{The presence of postoperative CTCs in glioma patients was a poor prognostic factor}

In this study, we initially enrolled 106 patients, and we have follow-up results for 79 patients, with the remaining patients lost contact. The median follow-up time from the end of the operation was 1210 days (range, 208-2140 days). A total of 55 patients (69.6\%) experienced disease progression, of which 51 patients died during follow-up (Additional file 1 Figure: S6A). As shown in Additional file 1 Figure: S6B and $C$, the higher the glioma grade was, the worse the OS and DFS $(p<$ 0.0001).

Furthermore, according to the results of CTC detection, the patients were divided into a CTC-positive group (CTCs $>1$ ) and a CTC-negative group (CTCs $\leq$ 1 ), and survival analysis was conducted for patients in different CTC groups before and after surgery. The median preoperative DFS times were as follows: 956 days (95\% CI, 775.6-1136.4) for the CTC-positive group and 1460 days for the CTC-negative group $(p=0.19)$, and the median OS time of preoperative patients was 1089 days (95\% CI=804.1-1373.9) for the CTC-positive group ( $p=$ 0.12) (Fig. 3A). However, postoperative CTCs were associated with significantly worse DFS and OS in patients (Fig. 3B). The median DFS was 858 days (95\% CI, 466.41249.6) for patients with positive CTC detection and 1454 days (95\% CI, 965.9-1942.2) for patients with negative CTC detection $(p=0.00045)$, and the median OS was 983 days (95\% CI, 636.7-1329.3) for patients with positive CTC detection and 1663 days (95\% CI, 1320.42005.6) for patients with negative CTC detection ( $p=$ 0.0011). Furthermore, for both OS and DFS in highgrade and low-grade glioma patients, there were no significant differences between the preoperative CTCpositive and CTC-negative groups (Fig. 3C, D). As shown in Fig. 3E and F, postoperative CTCs were significantly associated with DFS and OS in both low-grade patients and high-grade patients. In the low-grade glioma group, the positive postoperative CTC status was significant (DFS, 1173 days, 95\% CI=725.6-1620.4, $p=$ 0.0048; OS, 1358 days, 95\% $\mathrm{CI}=1317.7-1398.3, p=$ $0.0025)$. In the high-grade glioma group, positive and negative postoperative CTC statuses were similar in terms of DFS (368 vs. 1076 days, $95 \% \mathrm{CI}=35.1-700.9$ vs. 647.8-1504.2, $p=0.00045$ ) and OS (473 vs. 1089 days, $95 \% \mathrm{CI}=192.3-753.7$ vs. $694.5-1483.5, p=0.0022)$. The results revealed that postoperative CTCs may be an independent, significant predictor of DFS and OS.

\section{CTCs that predicted poor prognosis were associated with} postoperative neutrophils

To explore the relationship between CTCs and the peripheral immune environment, we further analyzed the correlation between the CTC number and the clinical blood examination data of 79 glioma patients with prognostic information (Additional file 4). Figure 4A shows significant differences in the proportions of lymphocytes and neutrophils between the CTC-negative group and the CTC-positive group $(p<0.0001)$, while there was no significant difference in the proportions of monocytes $(p=0.45)$. The correlation between CTCs and peripheral immune cells was further analyzed. Overall, in the 79 glioma patients after surgery, CTCs were significantly negatively correlated with the proportion of lymphocytes $(r=-0.62, p<0.0001)$ and significantly positively correlated with the proportion of neutrophils $(r=0.64, p<$ $0.0001)$, but no correlation was found with the proportion of monocytes $(r=-0.18, p=0.11$ ) (Fig. 4B-D). Heatmaps were constructed to show the correlation between the number of postoperative CTCs in the postoperative positive CTC group, the postoperative negative CTC group, and the 79 glioma patients with the clinical blood examination data (Fig. 4E-G). The results of the postoperative CTC-positive group were consistent with the overall correlations, and the postoperative CTC count was positively correlated with neutrophils in CTC-positive patients $(r=0.61, p<0.0001)$. The postoperative CTC-negative group showed no correlations between CTCs and the lymphocyte proportion, neutrophil proportion, or monocyte proportion (Additional file 1: Figure S7). Therefore, the level of postoperative CTCs, which predicts a poor prognosis after surgery, may be associated with neutrophils.

\section{CTCs are closely related to the activated neutrophil- associated microenvironment of immunity in glioma patients}

To verify the correlation between CTCs and the peripheral immune environment, especially neutrophils, RNA sequencing, and TBCD were performed simultaneously using the peripheral blood of 31 glioma patients after surgery. According to the test threshold specified above, the abovementioned 31 patients were divided into a CTC-positive group $(n=9)$ and a CTC-negative group $(n=22)$ according to the number of CTCs. We first used the EPIC algorithm to predict the cell components of peripheral blood immune cells in different patients, with 15,258 gene results obtained by RNA sequencing (Fig. $4 \mathrm{H})$. As shown in Fig. 4I and G, the proportion of neutrophils in the CTC-positive group was significantly higher than that in the CTC-negative group $(p<0.0001)$, while the other immune cells in the CTC-negative group either were significantly higher than those in the CTC- 


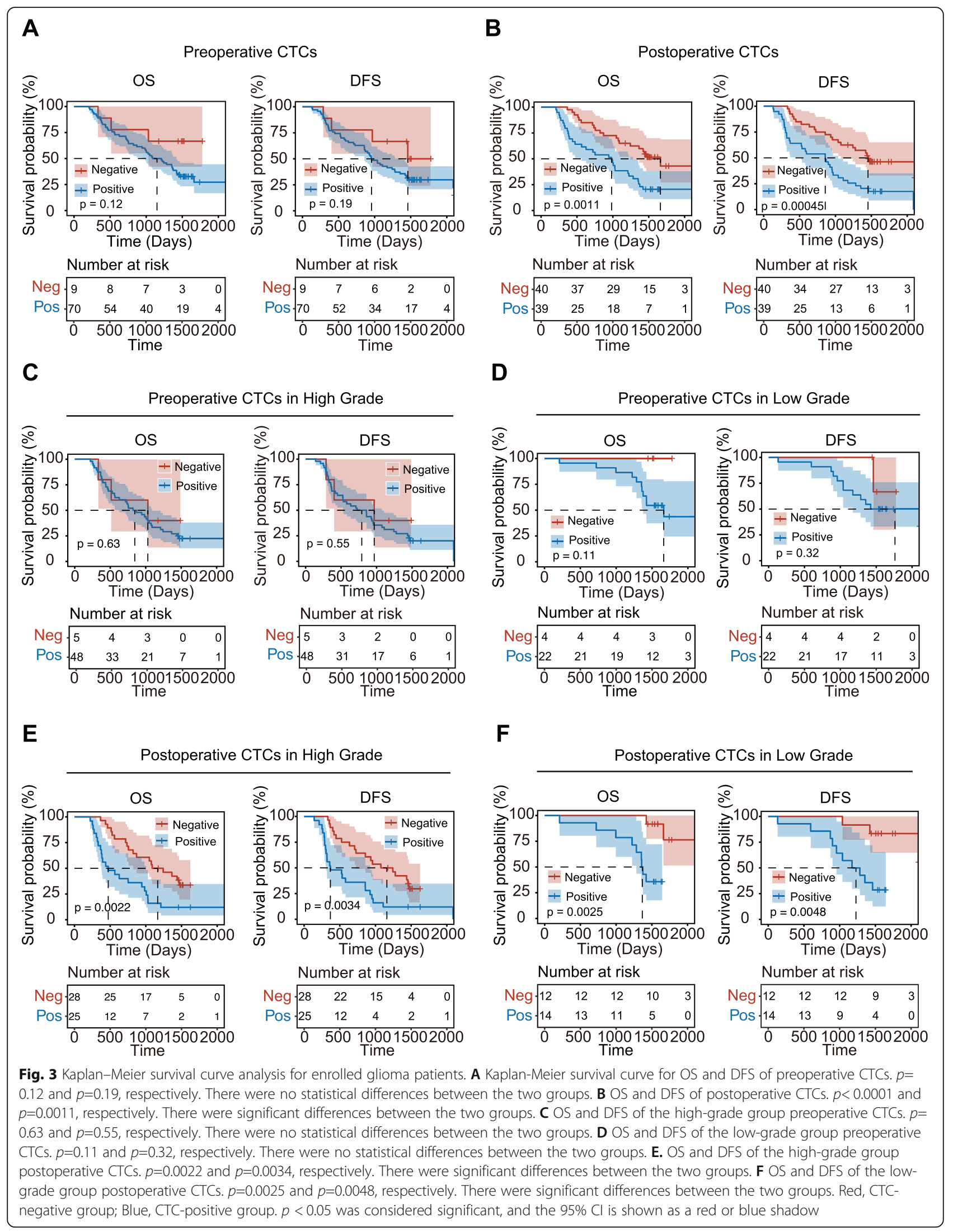




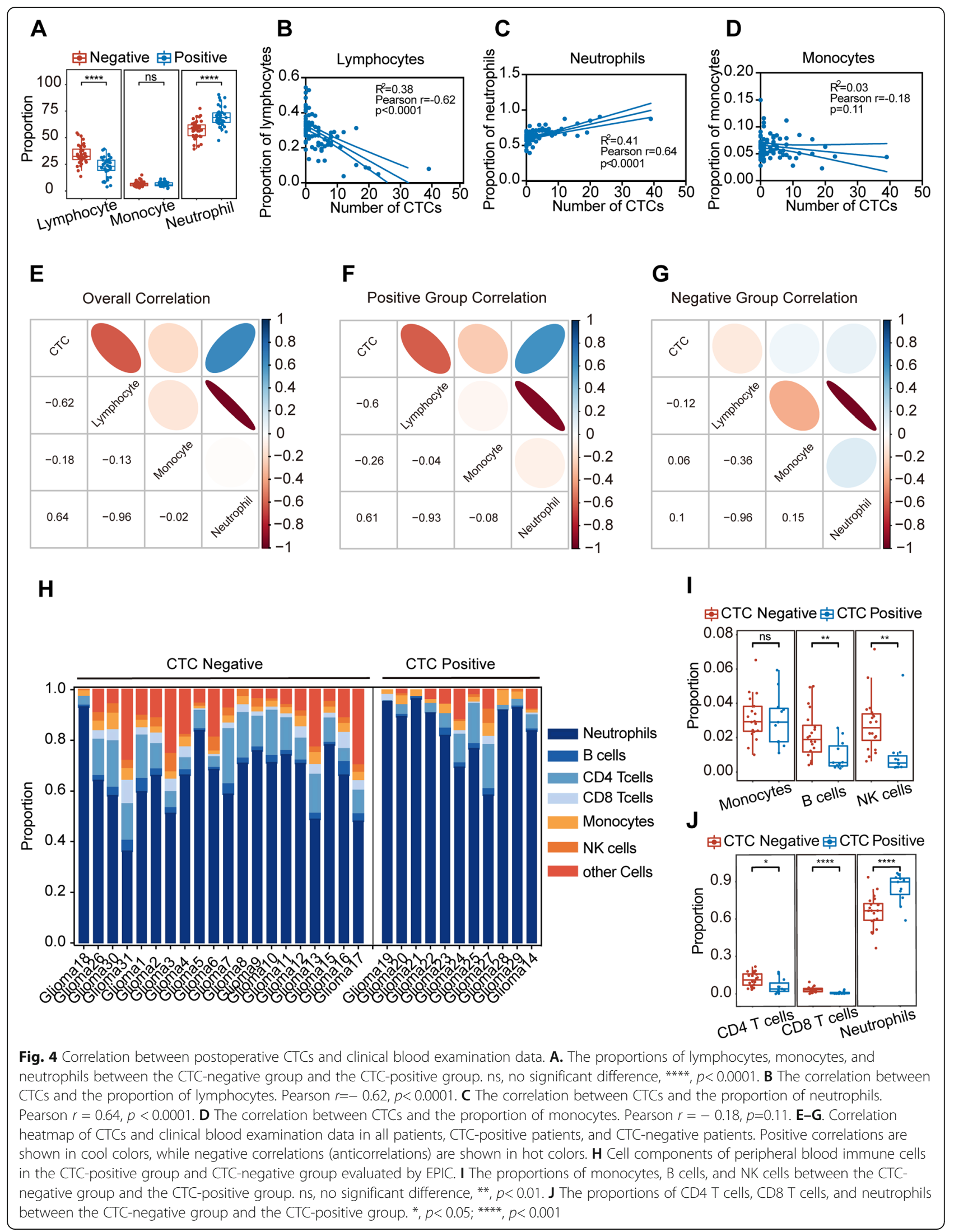


positive group or were not different (monocytes). Samples with higher CTC counts mainly showed high expression levels of neutrophils, while samples with low CTC counts mainly showed high expression levels of B cells, CD4 T cells, CD8 T cells, monocytes, and NK cells. The results showed that the correlation between the proportion of peripheral blood immune cells evaluated by sequencing results and CTCs was consistent with the correlation between the results of postoperative routine blood examination, and there was a significant positive correlation between CTCs and neutrophils in postoperative peripheral blood.

We further explored the relationship between the leukocyte transcriptome and CTC counts. Using 1729 significantly differentially expressed genes $(p<0.05$, $\mid \log 2$-fold change $\mid>1.5$ ) (Fig. 5A), we observed an overall difference in the peripheral blood of the positive and negative groups of glioma patients. PCA was performed in the two groups of glioma patients and the normal group (Fig. 5B). There were differences in the overall differential genes in peripheral blood among the three groups. Next, we identified 455 immunity-correlated genes [18] from 5416 CTC count-related genes (|Cor $\mid>$ $0.4, p<0.05)$. The PCA plots showed that the glioma high CTC group was significantly different from the low CTC group in the gene expression profile that was correlated with CTCs, while the negative CTC group was similar to the normal control group (Fig. 5C). The heatmap (Fig. 5D) shows significant differences between the 171 relevant genes in the positive and negative CTC groups $(\mid$ Cor $\mid>0.4, p<0.05)$. The results suggested that the postoperative CTC level was closely related to the postoperative immune status of patients.

When total RNA in the peripheral blood of glioma patients was analyzed by DESeq2, a volcano plot of the immune-related differential genes between the CTCpositive group and the CTC-negative group was obtained (Additional file 1: Figure S8A). Pathway and process enrichment analysis of the differentially expressed genes showed that the genes highly expressed in the CTC-positive group $(p<0.05, \log 2$-fold change $>1$, $n=200)$ correlated with the positive regulation of biological processes such as neutrophil degranulation, neutrophil activation, and neutrophil-mediated immunity (Fig. 5E, Additional file 5). In the CTC-negative group, the highly expressed genes $(n=252)$ were mainly associated with $\mathrm{T}$ cell activation, lymphocyte differentiation and proliferation, and $\mathrm{T}$ cell-mediated immune response (Fig. 5E, Additional file 6). The results indicated that CTCs were positively correlated with peripheral innate immune responses, especially the activation of neutrophils and the generation of neutrophil extracellular traps (NETs), but negatively correlated with the cytotoxic response, especially the $\mathrm{T}$ cell cytotoxic response. We speculated that CTC levels in glioma patients might be related to macroimmunity in the peripheral blood of patients.

To annotate the functions enriched in the 337 genes of interest that positively correlated with CTC levels (Cor> 0.4), Gene Ontology (GO) analysis was performed. The results showed that CTCs positively correlated with the 10 most significantly enriched biological process GO terms and were also associated with neutrophilmediated immune response, particularly neutrophil activation and degranulation (Fig. 6A, B). In the gene set enrichment analysis (GSEA), CTC count was regarded as a continuous variable. WBCs in patients with higher CTC counts showed a similar trend to the downregulated response of CD4 $\mathrm{T}$ cells/B cells instead of myeloid cells (Fig. 6C). The upregulation response of CD4 T cells instead of myeloid cells presented a negative correlation with CTCs (Additional file 1 Figure: S8B and C). Moreover, with a hallmark gene set (Fig. 6D), WBCs in patients with higher CTC counts were activated by TNF- $\alpha$ signaling via NF- $\mathrm{kB}$ and hypoxia (Fig. 6E). The activation of these mechanisms can promote the activation of neutrophils, particularly the neutrophil degranulation process, and these changes are consistent with CTC levels. In addition, neutrophilic proteins were analyzed to determine the relationship between NETs formation and tumor progression. GSEA was used to compare the relationship between NETs-related proteins and high and low glioma levels in the CTCs group and showed a difference in tumor transcriptome data between immune-related genes and NETs-related genes (Fig. 6F, Additional file 7). A volcano map of RNA expression based on NETS-related genes (Fig. 6G) is shown in the specific high-low subsets, indicating that patients with elevated CTC levels continued to stimulate increased levels of NETs expression. It can be speculated that the reconstruction of the macroimmune environment by tumor reduction in glioma patients is closely related to CTCs and that the reduction in the macroimmune inflammatory environment can achieve a better prognosis.

\section{Discussion}

In recent years, with in-depth research on the mechanism of tumor metastasis, great progress has been made in the use of CTC detection as a new noninvasive diagnostic method. Although the systemic metastasis of glioma is very rare, some studies have reported the successful isolation and identification of glioma CTCs from peripheral blood [20-28]. The presence of CTCs in gliomas is a common phenomenon, although the reported detection rates vary to a certain extent $(20-80 \%)$. However, the most prominent difference between central nervous system tumors and epidermal malignant tumors is the lack of EpCAM expression, which makes the 
A

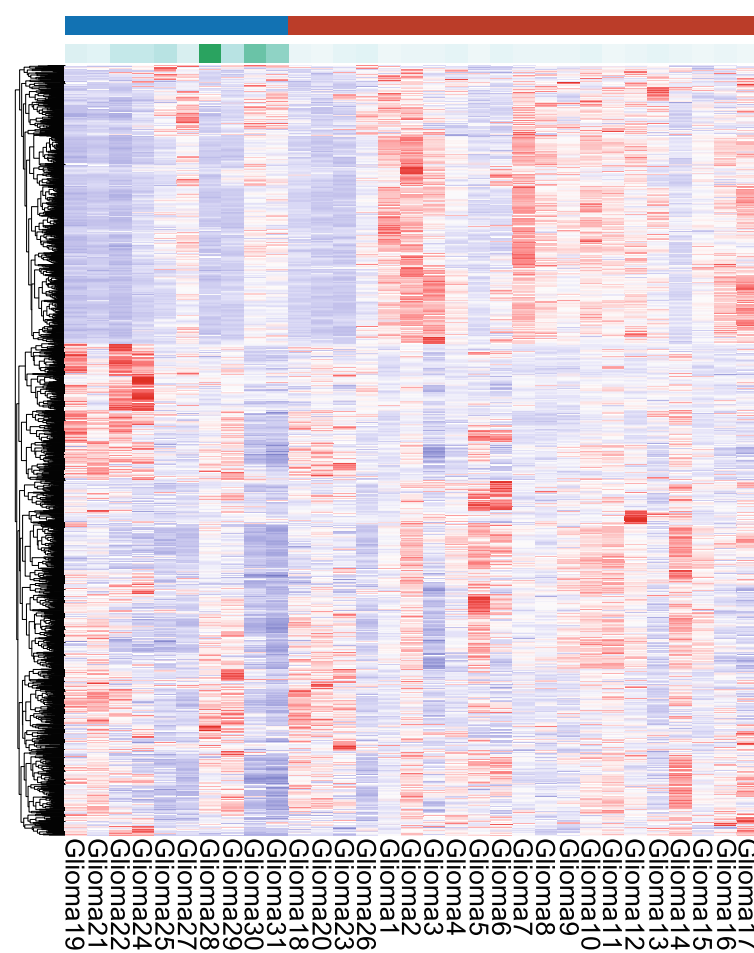

D

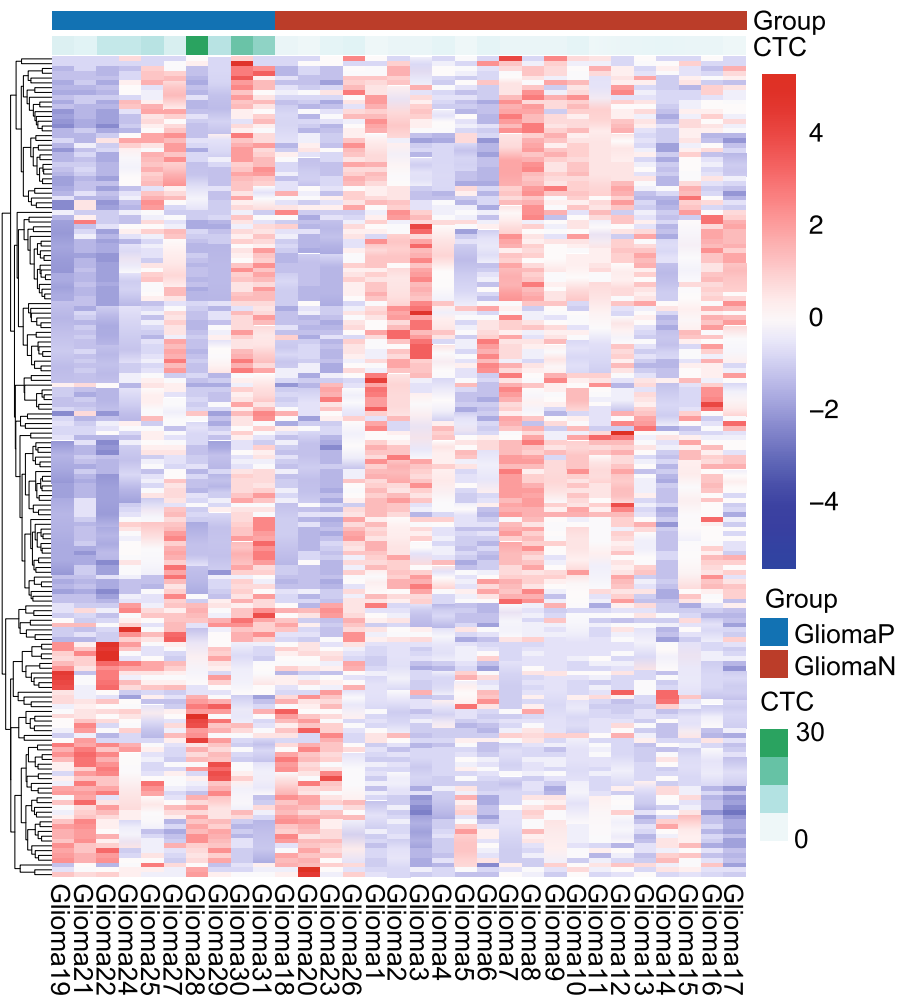

B

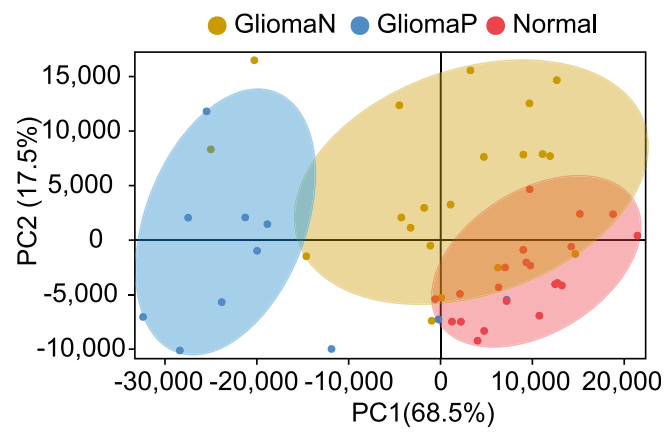

\section{C}

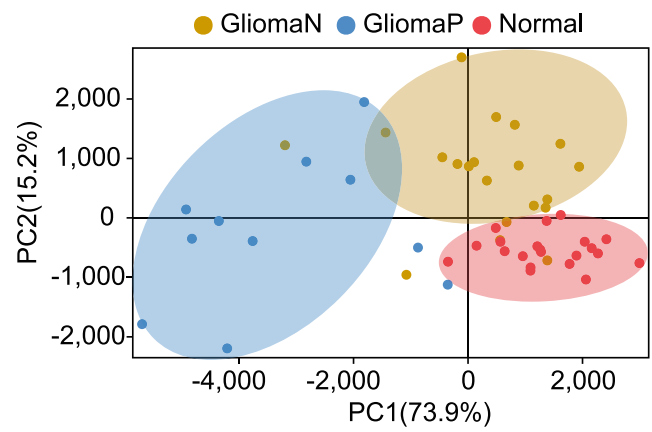

E

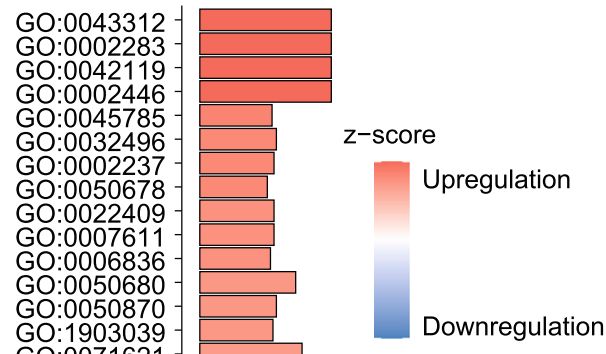

GO:1903039

GO:00071621

GO:0015718

GO:1901654

GO:0010633

GO:0001937

GO:0045058

GO:0033077

GO:0002706

GO:0046631-

GO:0002703

GO:0070663

GO:0046651

GO:0032943

GO:0050863

GO:0002460

GO:0030217

GO:0002696

G:0050867

GO:0050867

GO:0070661

GO:0002449

GO:0030098

GO:0042110

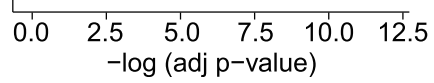

Fig. 5 (See legend on next page.) 
(See figure on previous page.)

Fig. 5 Relationship between postoperative CTC count and macroimmunity in peripheral blood. A Gene heatmap of 1729 significantly differentially expressed genes ( $p<0.05$, |log2-fold change|> 1.5) in 31 glioma patients. GliomaP: CTC-positive group, GliomaN: CTC-negative group. B Principal component analysis (PCA) of total genes for the CTC-positive group, CTC-negative group, and normal group. Red contour area: normal group, blue contour area: CTC-positive group, yellow contour area: CTC-negative group. C PCA of 455 immune-correlated genes with the CTC count for the CTC-positive group, CTC-negative group, and normal group. Red contour area: normal group, blue contour area: CTC-positive group, yellow contour area: CTC-negative group. D Gene heatmap of 171 immune-correlated genes (|Cor $\mid>0.4, p<0.05)$ in 31 glioma patients. GliomaP: CTC-positive group, GliomaN: CTC-negative group. E The most significant Gene Ontology (GO) terms enriched in the genes of interest related to CTC counts. The color of the bar indicates the $z$-score. Upregulation (red, positive $z$-score), downregulation (blue, negative $z$ score).

detection of glioma CTCs challenging and requires other strategies [27].

In this study, an hTERT-based detection method for circulating tumor cells in glioma patients was established. It has been reported that hTERT expression is increased in commonly used glioma cell lines (U251, U373, and U87) as well as in clinicopathological sections $[25,29]$. Based on previous studies, anti-CD45 antibodies can be used to reduce the background of CTC detection and to effectively reduce false positives in CTC detection [30]. Here, for 52 healthy individuals and 106 patients with glioma, the TBCD method was used for glioma sample (ROC) curve analysis. The participants who provided each of the 4-ml blood samples had $>1$ under the threshold of CTC, and the sensitivity of the difference between glioma patients and healthy individuals reached 0.830 and 0.865 , respectively, showing that the detection method showed very high efficiency.

Compared with other CTC detection methods, the CTC detection method for glioma based on the hTERT promoter has several advantages. First, it does not depend on the expression of tumor cell surface markers, such as EpCAM and GFAP; thus, the CTC tracer is independent of the expression levels of cell surface markers and specific molecular types. Second, since the vector requires infection and replication in active cancer cells to obtain GFP expression, this method labels only viable cells, thus obtaining more reliable CTC assessment results. Although studies have reported high hTERT expression in glioma using an adenovirus vector tracer [25], CTCs can be isolated via our strategy by flow cytometry and enable a further single-cell molecular analysis of informatics analyses to determine more accurate individualized treatment measures for patients.

At present, the reported studies on CTCs of glioma focus on GBM, while there are few studies on other pathological types $[20,27]$. In this study, the total detection rate of CTCs in glioma patients was $83.02 \%(n=88 /$ 106), which was the highest reported to date, and CTCs of different pathological subtypes were widely present in peripheral blood regardless of the degree of malignancy. This finding suggests that the presence of CTCs is a common feature of gliomas and is not unique to GBM. In the traditional monitoring of clinical response and glioma indicators, pseudoprogression or radiation necrosis may affect treatment judgment and lead to overtreatment [31, 32]. Because CTCs are widely present in peripheral blood, the number of CTCs can indirectly reflect the number and tumor load of intracranial tumor cells. Our results showed that at the 52-month follow-up after surgical treatment, there was a significant positive correlation between postoperative CTC levels and glioma DFS and OS. These results suggest that the CTCs detected by the TBCD assay are independent of the tumor characteristics of the patients and can be used as a supplement independent of other clinical and prognostic indicators.

Subsequently, we analyzed the relationship between systemic immune status and CTCs. In this study, we focused for the first time on CTCs associated with the peripheral immune system as a factor for poor prognosis in glioma. Until now, little has been known about the mechanism by which CTCs rely on the circulatory system to escape the killing attacks of various immune cells, migrate or return to their original sites, and implant there. A recent study has shown that neutrophils are the main white blood cells that interact with CTCs in mouse models and patients [33]. An increasing number of studies have shown that neutrophils play a role in all stages of tumor progression [34, 35]. Allen et al. revealed the remodeling effect of the tumor on systemic immunity through studies on a variety of animal models [36]. Among them, SB28 glioblastoma, located in the brain, has a greater impact on systemic immunity than other tumors. Therefore, through the analysis of the systemic immunity of glioma patients and the correlation between systemic immunity and CTCs, the ability of clinical evaluation and prediction can be further improved.

Although there has been some understanding of the relationship between CTCs and systemic immunity, there are few reports on the association of neutrophils or systemic immune status with glioma CTCs. Our study data showed that postoperative CTC status highly correlated with the overall immunity of the body. On the one hand, the peripheral immune state is characterized by the promotion of the secretion of the inflammatory cytokine TNF- $\alpha$ through neutrophil degranulation or activity enhancement, and TNF- $\alpha$ is associated with the 


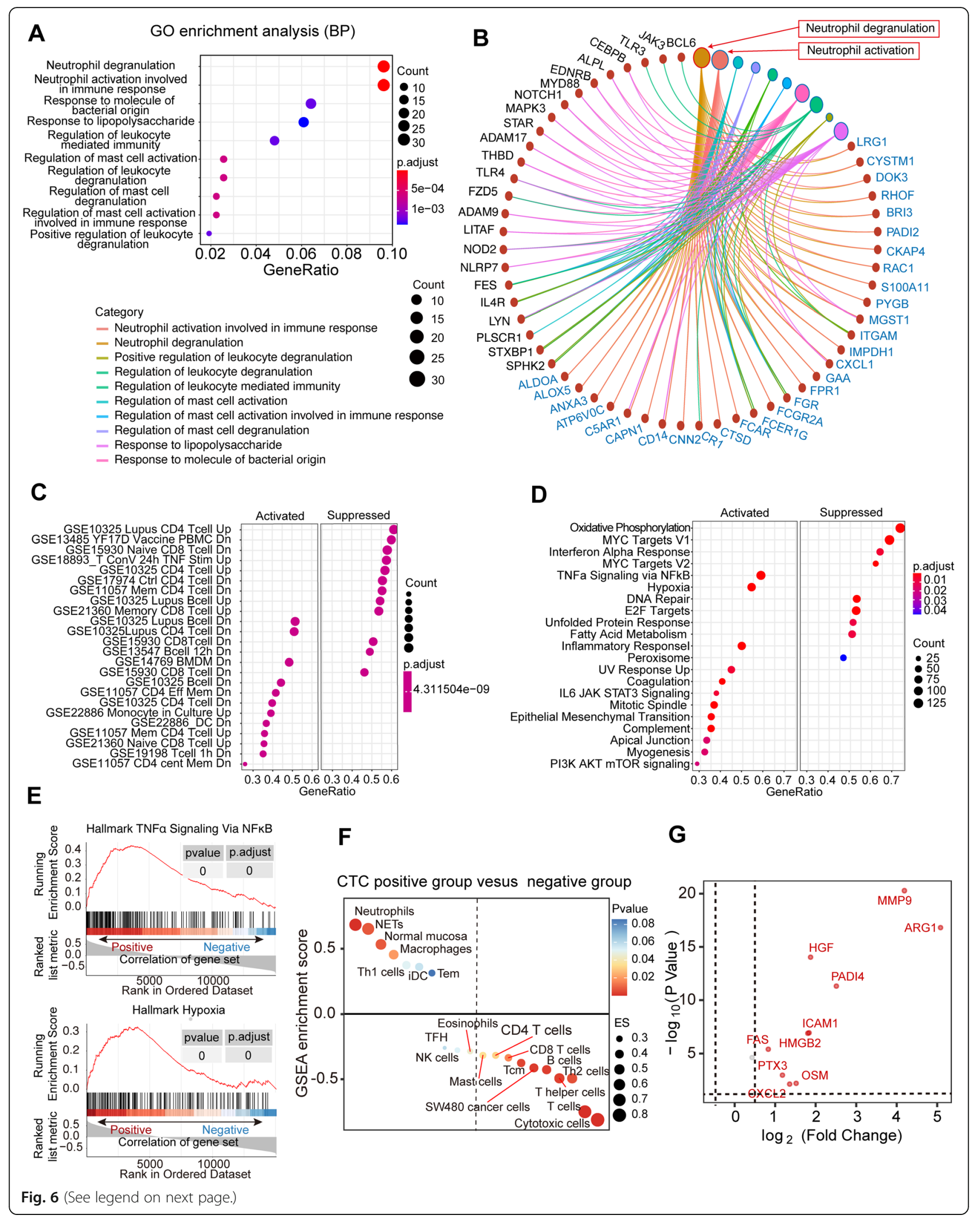


(See figure on previous page.)

Fig. 6 Gene profiles positively correlated with CTC count. A The 10 most significant Gene Ontology (GO) terms enriched in the genes of interest. GeneRatio is the percentage of total differential genes in the given $\mathrm{GO}$ term. Count is the number of total differential genes in the given GO term. $\mathbf{B}$ The maximal subnetwork constructed with the genes of interest. The pink nodes represent neutrophil activation, and the second-largest brown node is neutrophil degranulation. The size and color of the edges indicate the coexpression values. Count is the number of total differential genes in the given GO term. $\mathbf{C}$. GSEA of glioma sample data ( $n=31$ ) for the c7 immunological gene sets downloaded from the Molecular Signatures Database (MSigDB). GeneRatio is the percentage of total differential genes in the given GO term. Count is the number of total differential genes in the given GO term. D. GSEA of glioma sample data ( $n=31$ ) using the hallmark v7.0 gene sets (MSigDB). GeneRatio is the percentage of total differential genes in the given GO term. Count is the number of total differential genes in the given GO term. E GSEA of the peripheral blood leukocyte RNA-seq dataset. Activated gene sets showed TNF-a signaling via NF$\mathrm{KB}(p$ value $<0.05)$ with a minimum enrichment score of 0.3 (upper image). Activated gene sets showed TNF-a signaling via hypoxia $(p$ value $<0.05)$ with a minimum enrichment score of 0.3 (bottom image). F The relationship between NET scores and gene sets in the CTC-positive and CTC-negative groups. G A volcano plot of NET-related differential genes was obtained between the CTC-positive group and the CTC-negative group

activation of neutrophil extracellular traps (NETs) in the inflammatory response [37, 38]. Regarding glioma patients, the level of CTCs and the expression of NETsrelated genes showed obvious convergence, and we thus infer that there is a close relationship between glioma CTCs and NETs, which is associated with a reduction in cell-killing ability, leading to immune escape and a poor prognosis. On the other hand, the analysis of RNA sequencing results also showed $\mathrm{T}$-cell-mediated immune cell damage and decreased lymphocyte immune regulation ability. Additionally, this study showed a correlation between hypoxia and CTCs in the systemic immune state. CTCs in the systemic immune state also face adaptive changes in the hypoxic environment. In the peripheral blood of glioma patients, CTCs significantly correlated with the increased expression of HIF-1 $\alpha$ in the systemic immune system. HIF-1 $\alpha$ may act as a protective factor for peripheral CTC survival, preventing apoptosis in the peripheral circulation of CTCs and thereby shortening the predicted OS times of patients [39].

Another interesting phenomenon in this study is that although CTCs can be detected in glioma patients both before and after surgery, the patient prognosis was not related to preoperative CTC levels but was significantly related to postoperative CTC levels. There are few studies on the correlation between preoperative CTCs and the prognosis of patients with glioma, but Lynch D. et al. also showed no correlation between CTCs and PFS or OS in a small cohort of patients with glioma [40]. We speculate that CTCs are likely to interact with the immune system, especially the inflammatory response, resulting in a poor patient prognosis. At the tumor site, the inflammatory response is a negative prognostic factor for gliomas [41, 42], and the release of inflammatory factors tends to accelerate glioma progression [43]. However, preoperative tumor-related factors in glioma patients, including pathological grade, lesion site, tumor location, mass size, and edema degree, did not affect inflammatory factors [44]. Therefore, although CTCs exist in glioma patients before surgery, they have no effect on the prognosis of glioma patients due to the systemic noninflammatory immune environment. These results suggest that the systemic immune status should also be considered in the clinical treatment of glioma patients and that targeted systemic immunomodulatory therapy, especially of neutrophils associated with NETs formation, may benefit patients clinically.

\section{Conclusions}

In conclusion, we propose an application to facilitate clinical detection and treatment monitoring for glioma patients by using the TBCD method. This method can stably and easily detect CTCs of a wide range of pathological types of glioma and has the potential to monitor the disease progression of glioma patients, predict prognosis and evaluate the efficacy of individualized treatment. In addition, our results also showed a correlation between CTCs and peripheral immune system immunity, which provides a new perspective for the treatment of glioma.

\section{Abbreviations}

CTCs: Circulating tumor cells; OS: Overall survival; DFS: Disease-free survival; Cl: Confidence interval; TBCD: Telomerase reverse transcriptase-based CTC detection method; NETs: Neutrophil extracellular traps

\section{Supplementary Information}

The online version contains supplementary material available at https://doi. org/10.1186/s12916-021-02138-7.

\section{Additional file 1. \\ Additional file 2. \\ Additional file 3. \\ Additional file 4. \\ Additional file 5 . \\ Additional file 6 . \\ Additional file 7.}

\section{Acknowledgements}

We thank the staff of the National Center for Protein Sciences Beijing (Peking University) and Ms. Fei Wang for her technical assistance with flow cytometry imaging. We also thank Diatech Co., technical support for the TBCD method. 


\section{Authors' contributions}

WZ and TCQ have contributed equally to this work. SJC, KTZ, and WZ conceived the study. BBL, KTZ, and WZ designed the study. WZ, TCQ, ZCY, $L Y Y$, and CYZ. performed the experiments. TCQ and SL collected the samples and clinical information. TCQ and WZ performed the data analysis. WZ and TCQ wrote the manuscript with input from all authors. LF and BLL contributed to the interpretation of the results. WZ and KTZ supervised the study. All authors provided critical review of the manuscript and approved its final version for submission.

\section{Funding}

This research was funded by the National Key R\&D Program of China (2019YFC1315702) and the National Natural Science Foundation of China (81472013).

\section{Availability of data and materials}

All data generated or analyzed during this study are included in this published article. The raw RNA sequencing data generated in this study are deposited in the Genome Sequence Archive at the BIG Data Center, Beijing Institute of Genomics (BIG), Chinese Academy of Sciences, under the accession number HRA001221 [https://bigd.big.ac.cn/gsa-human/browse/ HRA001221].

\section{Declarations}

\section{Ethics approval and consent to participate}

This study was carried out in accordance with the recommendations of the Ethics Committee of the Cancer Institute and Hospital of the Chinese Academy of Medical Sciences with written informed consent from all subjects.

\section{Consent for publication}

Not applicable.

\section{Competing interests}

All authors declare that they have no competing interests.

\section{Author details}

'Department of Immunology, National Cancer Center/National Clinical Research Center for Cancer/Cancer Hospital, Chinese Academy of Medical Sciences and Peking Union Medical College, Beijing 100021, China. ${ }^{2}$ State Key Laboratory of Molecular Oncology, Department of Etiology and Carcinogenesis, National Cancer Center/National Clinical Research Center for Cancer/Cancer Hospital, Chinese Academy of Medical Sciences and Peking Union Medical College, Beijing 100021, China. ${ }^{3}$ Lung Cancer Center, West China Hospital, Sichuan University, Chengdu 610041, China. ${ }^{4}$ Chongqing Diatech Biotechnological Limited Company, Chongqing 400020, China. ${ }^{5}$ Department of Neurosurgery, Beijing Tiantan Hospital, Capital Medical University, Beijing, China. ${ }^{6}$ National Clinical Research Center for Neurological Diseases, Center of Brain Tumor, Beijing Institute for Brain Disorders and Beijing Key Laboratory of Brain Tumor, Beijing 100070, China. "National "111" Center for Cellular Regulation and Molecular Pharmaceutics, Key Laboratory of Fermentation Engineering (Ministry of Education), Hubei Provincial Cooperative Innovation Center of Industrial Fermentation, College of Bioengineering, Hubei University of Technology, Wuhan 430068, China.

Received: 22 July 2021 Accepted: 20 September 2021 Published online: 12 November 2021

\section{References}

1. Zhang H, Liao J, Zhang X, Zhao E, Liang X, Luo S, et al. Sex difference of mutation clonality in diffuse glioma evolution. Neuro Oncol. 2019;21(2):201213. https://doi.org/https://doi.org/10.1093/neuonc/noy154.

2. Chinot OL, Wick W, Mason W, Henriksson R, Saran F, Nishikawa R, et al. Bevacizumab plus radiotherapy-temozolomide for newly diagnosed glioblastoma. N Engl J Med. 2014;370(8):709-722. https://doi.org/https://doi. org/10.1056/NEJMoa1308345.

3. Miyauchi JT, Tsirka SE. Advances in immunotherapeutic research for glioma therapy. J Neurol. 2018;265(4):741-756. https://doi.org/https://doi.org/10.1 007/s00415-017-8695-5.
4. Stupp R, Mason WP, van den Bent MJ, Weller M, Fisher B, Taphoorn MJ, et al. Radiotherapy plus concomitant and adjuvant temozolomide for glioblastoma. N Engl J Med. 2005;352(10):987-996. https://doi.org/https://doi. org/10.1056/NEJMoa043330.

5. Stupp R, Hegi ME, Gorlia T, Erridge SC, Perry J, Hong YK, et al. Cilengitide combined with standard treatment for patients with newly diagnosed glioblastoma with methylated MGMT promoter (CENTRIC EORTC 2607122072 study): a multicentre, randomised, open-label, phase 3 trial. Lancet Oncol. 2014;15(10):1100-1108. https://doi.org/https://doi.org/10.1016/S14 70-2045(14)70379-1.

6. Lapointe S, Perry A, Butowski NA. Primary brain tumours in adults. Lancet. 2018;392(10145):432-446. https://doi.org/https://doi.org/10.1016/50140-673 6(18)30990-5

7. Bi J, Chowdhry S, Wu S. Altered cellular metabolism in gliomas - an emerging landscape of actionable co-dependency targets. Nat Rev Cancer. 2020;20(1):57-70. https://doi.org/https://doi.org/10.1038/s41568-019-0226-5.

8. Touat M, Duran-Peña A, Alentorn A, Lacroix L, Massard C, Idbaih A. Emerging circulating biomarkers in glioblastoma: promises and challenges. Expert Rev Mol Diagn. 2015;15(10):1311-1323. https://doi.org/https://doi. org/10.1586/14737159.2015.1087315.

9. Figueroa JM, Carter BS. Detection of glioblastoma in biofluids. J Neurosurg. 2018;129(2):334-340. https://doi.org/https://doi.org/10.3171/2017.3.JNS162280.

10. Zhang W, Bao L, Yang S, Qian Z, Dong M, Yin L, et al. Tumor-selective replication herpes simplex virus-based technology significantly improves clinical detection and prognostication of viable circulating tumor cells. Oncotarget. 2016;7(26):39768-39783. https://doi.org/https://doi.org/10.18632/ oncotarget.9465.

11. Zhang W, Duan X, Zhang Z, Yang Z, Zhao C, Liang C, Liu Z, Cheng S, Zhang K. Combination of $C T$ and telomerase ${ }^{+}$circulating tumor cells improves diagnosis of small pulmonary nodules. JCI Insight. 2021;6(11):e148182. https://doi: https://doi.org/10.1172/jci.insight.148182.

12. Otasek D, Morris JH, Bouças J, Pico AR, Demchak B. Cytoscape Automation: empowering workflow-based network analysis. Genome Biol. 2019;20(1):185. https://doi.org/https://doi.org/10.1186/s13059-019-1758-4.

13. UniProt Consortium. UniProt: the universal protein knowledgebase in 2021. Nucleic Acids Res. 2021;49(D1):D480-D489. https://doi: https://doi.org/10.1 093/nar/gkaa1 100

14. Zhou Y, Zhou B, Pache L, Chang M, Khodabakhshi AH, Tanaseichuk O, et al. Metascape provides a biologist-oriented resource for the analysis of systems-level datasets. Nat Commun. 2019;10(1):1523. https://doi.org/10. 1038/s41467-019-09234-6.

15. Subramanian A, Tamayo P, Mootha VK, Mukherjee S, Ebert BL, Gillette MA, et al. Gene set enrichment analysis: a knowledge-based approach for interpreting genome-wide expression profiles. Proc Natl Acad Sci U S A. 2005;102(43):15545-15550. https://doi.org/https://doi.org/10.1073/pnas. 0506580102.

16. Liberzon A, Subramanian A, Pinchback R, Thorvaldsdóttir H, Tamayo P, Mesirov JP. Molecular signatures database (MSigDB) 3.0. Bioinformatics. 2011;27(12):1739-1740. https://doi.org/https://doi.org/10.1093/bioinforma tics/btr260.

17. Racle J, de Jonge $K$, Baumgaertner $P$, Speiser $D E$, Gfeller $D$. Simultaneous enumeration of cancer and immune cell types from bulk tumor gene expression data. Elife. 2017;6:e26476. https://doi.org/https://doi.org/10.7554/eLife.26476.

18. Bindea G, Mlecnik B, Tosolini M, Kirilovsky A, Waldner M, Obenauf AC, et al. Spatiotemporal dynamics of intratumoral immune cells reveal the immune landscape in human cancer. Immunity. 2013;39(4):782-795. https://doi.org/ https://doi.org/10.1016/j.immuni.2013.10.003.

19. Garzia L, Kijima N, Morrissy AS, De Antonellis P, Guerreiro-Stucklin A, Holgado BL, et al. A Hematogenous Route for Medulloblastoma Leptomeningeal Metastases. Cell. 2018;173(6):1549. https://doi.org/https:// doi.org/10.1016/j.cell.2018.01.038.

20. Liu T, Xu H, Huang M, Ma W, Saxena D, Lustig RA, et al. Circulating Glioma Cells Exhibit Stem Cell-like Properties. Cancer Res. 2018;78(23):6632-6642. https://doi.org/https://doi.org/10.1158/0008-5472.CAN-18-0650.

21. Müller C, Holtschmidt J, Auer M, Heitzer E, Lamszus K, Schulte A, et al. Hematogenous dissemination of glioblastoma multiforme. Sci Transl Med. 2014; 6(247):247ra101. https:/doi.org/https://doi.org/10.1126/scitranslmed.3009095.

22. Sullivan JP, Nahed BV, Madden MW, Oliveira SM, Springer S, Bhere D, et al, Brain tumor cells in circulation are enriched for mesenchymal gene expression. Cancer Discov. 2014;4(11):1299-1309. https://doi.org/https://doi. org/10.1158/2159-8290.CD-14-0471. 
23. de Bono JS, Scher HI, Montgomery RB, Parker C, Miller MC, Tissing H, et al. Circulating tumor cells predict survival benefit from treatment in metastatic castration-resistant prostate cancer. Clin Cancer Res. 2008;14(19):6302-6309. https://doi.org/https://doi.org/10.1158/1078-0432.CCR-08-0872.

24. Bang-Christensen SR, Pedersen RS, Pereira MA, Clausen TM, Løppke C, Sand NT, et al. Capture and Detection of Circulating Glioma Cells Using the Recombinant VAR2CSA Malaria Protein. Cells. 2019;8(9):998. https://doi.org/ https://doi.org/10.3390/cells8090998.

25. Macarthur KM, Kao GD, Chandrasekaran S, Alonso-Basanta M, Chapman C, Lustig RA, et al. Detection of brain tumor cells in the peripheral blood by a telomerase promoter-based assay. Cancer Res. 2014;74(8):2152-2159. https:// doi.org/https://doi.org/10.1158/0008-5472.CAN-13-0813.

26. Krol I, Castro-Giner F, Maurer M, Gkountela S, Szczerba BM, Scherrer R, et al. Detection of circulating tumour cell clusters in human glioblastoma. $\mathrm{Br} \mathrm{J}$ Cancer. 2018;119(4):487-491. https://doi.org/https://doi.org/10.1038/s41416018-0186-7.

27. Sareen H, Garrett C, Lynch D, Powter B, Brungs D, Cooper A, et al. The Role of Liquid Biopsies in Detecting Molecular Tumor Biomarkers in Brain Cancer Patients. Cancers (Basel). 2020;12(7):1831. https://doi.org/https://doi.org/1 $0.3390 /$ cancers12071831.

28. Adamczyk LA, Williams H, Frankow A, Ellis HP, Haynes HR, Perks C, et al. Current Understanding of Circulating Tumor Cells - Potential Value in Malignancies of the Central Nervous System. Front Neurol. 2015;6:174. https://doi.org/https://doi.org/10.3389/fneur.2015.00174.

29. Komata $T$, Kondo $Y$, Kanzawa $T$, Ito H, Hirohata $S$, Koga $S$, et al. Caspase-8 gene therapy using the human telomerase reverse transcriptase promoter for malignant glioma cells. Hum Gene Ther. 2002;13(9):1015-1025. https:// doi.org/https://doi.org/10.1089/104303402753812421.

30. Sharma S, Zhuang R, Long M, Pavlovic M, Kang Y, llyas A, et al. Circulating tumor cell isolation, culture, and downstream molecular analysis. Biotechnol Adv. 2018;36(4):1063-1078. https://doi.org/https://doi.org/10.1016/j.biotecha dv.2018.03.007.

31. Brandes AA, Franceschi E, Tosoni A, Blatt V, Pession A, Tallini G, et al. MGMT promoter methylation status can predict the incidence and outcome of pseudoprogression after concomitant radiochemotherapy in newly diagnosed glioblastoma patients. J Clin Oncol. 2008;26(13):2192-2197. https://doi.org/https://doi.org/10.1200/JCO.2007.14.8163.

32. Clarke JL, Chang S. Pseudoprogression and pseudoresponse: challenges in brain tumor imaging. Curr Neurol Neurosci Rep. 2009;9(3):241-246. https:// doi.org/https://doi.org/10.1007/s11910-009-0035-4.

33. Szczerba BM, Castro-Giner F, Vetter M, Krol I, Gkountela S, Landin J, et al. Neutrophils escort circulating tumour cells to enable cell cycle progression. Nature. 2019;566(7745):553-557. https://doi.org/https://doi.org/10.1007/s1191 0-009-0035-4.

34. Mollinedo F. Neutrophil Degranulation, Plasticity, and Cancer Metastasis. Trends Immunol. 2019;40(3):228-242. https://doi.org/https://doi.org/10.1016/ j.it.2019.01.006.

35. Jaillon S, Ponzetta A, Di Mitri D, Santoni A, Bonecchi R, Mantovani A. Neutrophil diversity and plasticity in tumour progression and therapy. Nat Rev Cancer. 2020;20(9):485-503. https://doi.org/https://doi.org/10.1038/s41 568-020-0281-y.

36. Allen BM, Hiam KJ, Burnett CE, Venida A, DeBarge R, Tenvooren I, et al. Systemic dysfunction and plasticity of the immune macroenvironment in cancer models. Nat Med. 2020;26(7):1125-1134. https://doi.org/https://doi. org/10.1038/s41591-020-0892-6.

37. Dinallo V, Marafini I, Di Fusco D, Laudisi F, Franzè E, Di Grazia A, et al. Neutrophil Extracellular Traps Sustain Inflammatory Signals in Ulcerative Colitis. J Crohns Colitis. 2019;13(6):772-784. https://doi.org/https://doi.org/1 0.1093/ecco-jcc/jjy215.

38. Carmona-Rivera C, Khaznadar SS, Shwin KW, Irizarry-Caro JA, O'Neil LJ, Liu Y, et al. Deficiency of adenosine deaminase 2 triggers adenosine-mediated NETosis and TNF production in patients with DADA2. Blood. 2019;134(4): 395-406. https://doi.org/10.1182/blood.2018892752.

39. Donato C, Kunz L, Castro-Giner F, Paasinen-Sohns A, Strittmatter K, Szczerba BM, et al. Hypoxia Triggers the Intravasation of Clustered Circulating Tumor Cells. Cell Rep. 2020;32(10):108105. https://doi.org/https://doi.org/10.1016/j. celrep.2020.108105.

40. Lynch D, Powter B, Po J.W, Cooper A, Garrett C, Koh E.-S, et al. Isolation of Circulating Tumor Cells from Glioblastoma Patients by Direct Immunomagnetic Targeting. Appl. Sci. 2020;10(9):3338. https://doi.org/ https://doi.org/10.3390/app10093338.
41. Ott M, Tomaszowski KH, Marisetty A, Kong LY, Wei J, et al. Profiling of patients with glioma reveals the dominant immunosuppressive axis is refractory to immune function restoration. JCI Insight. 2020;5(17):e134386. https://doi.org/https://doi.org/10.1172/jci.insight.134386.

42. Marinari E, Allard M, Gustave R, Widmer V, Philippin G, Merkler D, et al. Inflammation and lymphocyte infiltration are associated with shorter survival in patients with high-grade glioma. Oncoimmunology. 2020;9(1): 1779990. https://doi.org/https://doi.org/10.1080/2162402X.2020.1779990.

43. De Boeck A, Ahn BY, D'Mello C, Lun X, Menon SV, Alshehri MM, et al. Glioma-derived IL-33 orchestrates an inflammatory brain tumor microenvironment that accelerates glioma progression. Nat Commun. 2020; 11(1):4997. https://doi.org/https://doi.org/10.1038/s41467-020-18569-4.

44. Song L, Quan X, Su L, Wang K, Wang H, Wu L, et al. Inflammation and behavioral symptoms in preoperational glioma patients: Is depression, anxiety, and cognitive impairment related to markers of systemic inflammation? Brain Behav. 2020;10(9):e01771. https://doi.org/https://doi. org/10.1002/brb3.1771.

\section{Publisher's Note}

Springer Nature remains neutral with regard to jurisdictional claims in published maps and institutional affiliations.
Ready to submit your research? Choose BMC and benefit from:

- fast, convenient online submission

- thorough peer review by experienced researchers in your field

- rapid publication on acceptance

- support for research data, including large and complex data types

- gold Open Access which fosters wider collaboration and increased citations

- maximum visibility for your research: over $100 \mathrm{M}$ website views per year

At BMC, research is always in progress.

Learn more biomedcentral.com/submissions 